\title{
Worsening of Essential Tremor After SARS-CoV-2 Infection
}

\author{
Massimiliano Passaretti ${ }^{1}$ - Alessandro De Biase ${ }^{1}$ - Giulia Paparella ${ }^{2} \cdot$ Luca Angelini $^{1} \cdot$ Antonio Cannavacciuolo $^{2}$. \\ Donato Colella ${ }^{1} \cdot$ Alfredo Berardelli $^{1,2} \cdot$ Matteo Bologna ${ }^{1,2}$
}

Accepted: 31 December 2021 / Published online: 6 January 2022

(c) The Author(s), under exclusive licence to Springer Science+Business Media, LLC, part of Springer Nature 2022

\section{Dear Editor,}

In 2019, acute respiratory syndrome coronavirus 2 (SARS$\mathrm{CoV}-2$ ) was identified as the causative agent of a cluster of respiratory infections, later named coronavirus disease 2019 (COVID-19) [1]. Various reports documented that patients with movement disorders, including Parkinson's disease, experienced a worsening of their symptoms, e.g., tremor, bradykinesia, and gait disturbances, after severe COVID19 [2-6], although definite conclusions on the COVID-19 impact could not be made in most cases [2-6]. In essential tremor (ET), the possible consequences of SARS-CoV-2 infection are uninvestigated. Here we discuss the case of an ET patient whose symptoms worsened after asymptomatic SARS-CoV-2 infection.

A 60-year-old right-handed man diagnosed with ET in 2015 complained of significant tremor worsening in July 2021. The patient's symptoms had started at the age of 17 years with action tremor of the upper limbs, which was slightly predominant on the left side and responsive to alcohol administration. The patient had not rest tremor. He reported a family history of tremor on the maternal side (both grandparents and three mother's siblings). In 2015, he started propranolol $40 \mathrm{mg}$ daily. A brain magnetic resonance imaging (MRI) performed in June 2017 documented millimetric left pons lacunes. From 2015 to 2021, no changes in tremor severity were documented and no therapy modification was needed. The patient was enrolled in a longitudinal research study on ET progression, for which he underwent comprehensive clinical and kinematic assessments in June 2015 (T1) and December 2020 (T2), with movement (FahnTolosa-Marin Tremor Rating Scale (FTMTRS), Movement

Matteo Bologna

matteo.bologna@uniroma1.it

1 Department of Human Neurosciences, Sapienza University of Rome, Viale dell'Università 30, 00185 Rome, Italy

2 Neuromed Institute IRCCS, Pozzilli, IS, Italy
Disorder Society Unified Parkinson's Disease Rating Scale (MDS-UPSRD)), psychiatric (Brief Psychiatric Rating Scale (BPRS); Clinical Global Impression (CGI); Hamilton Anxiety Rating Scale (HAM-A); Hamilton Depression Rating Scale (HAM-D)), and cognitive functions (Montreal Cognitive Assessment (MoCA), Mini-Mental State Examination (MMSE)) evaluated by standardized scales. The assessment also included kinematic analysis of upper limb tremor as detailed in [7]. Briefly, kinematic recordings were performed using an optoelectronic system (SMART motion system, BTS Engineering, Italy) consisting of three infrared cameras (sampling rate of $120 \mathrm{~Hz}$ ) and reflective markers of negligible weight taped to the subject's arms and trunk [7]. Upper limb postural tremor of was recorded: (i) with the arms outstretched in front of the chest (posture 1), and (ii) with the arms flexed at the elbows, i.e., lateral "wing beating" posture (posture 2). Upper limb kinetic tremor was recorded during a "pointing task," in which the subject was asked to repetitively move his index finger from their nose to a reflective target fixed on a heavy support approximately $15 \mathrm{~cm}$ above the table at sternal height and at approximately $2 / 3$ arm distance. Rest tremor of the upper limbs was recorded while the patient sat comfortably on a chair facing the cameras, with his arms laying on a desk in front of him. Tremor analysis was performed using dedicated software (SMART Analyzer, BTS Engineering, Italy) [7]. Written informed consent was obtained.

In April 2021, the patient was diagnosed with asymptomatic SARS-CoV-2 infection, confirmed by four consecutive polymerase-chain-reaction nasopharyngeal swab test, during a 28-day period. Three weeks later, he noticed tremor worsening that persisted over time. In July 2021 (T3), an aggravation of postural and kinetic upper limb tremor was clinically present (Fig. 1). No other neurological signs were observed. Psychiatric and cognitive functions did not change as compared to previous evaluations. Brain MRI and laboratory exams did not depict any appreciable changes. The patient underwent a further kinematic assessment, which documented an increase in postural tremor amplitude as 


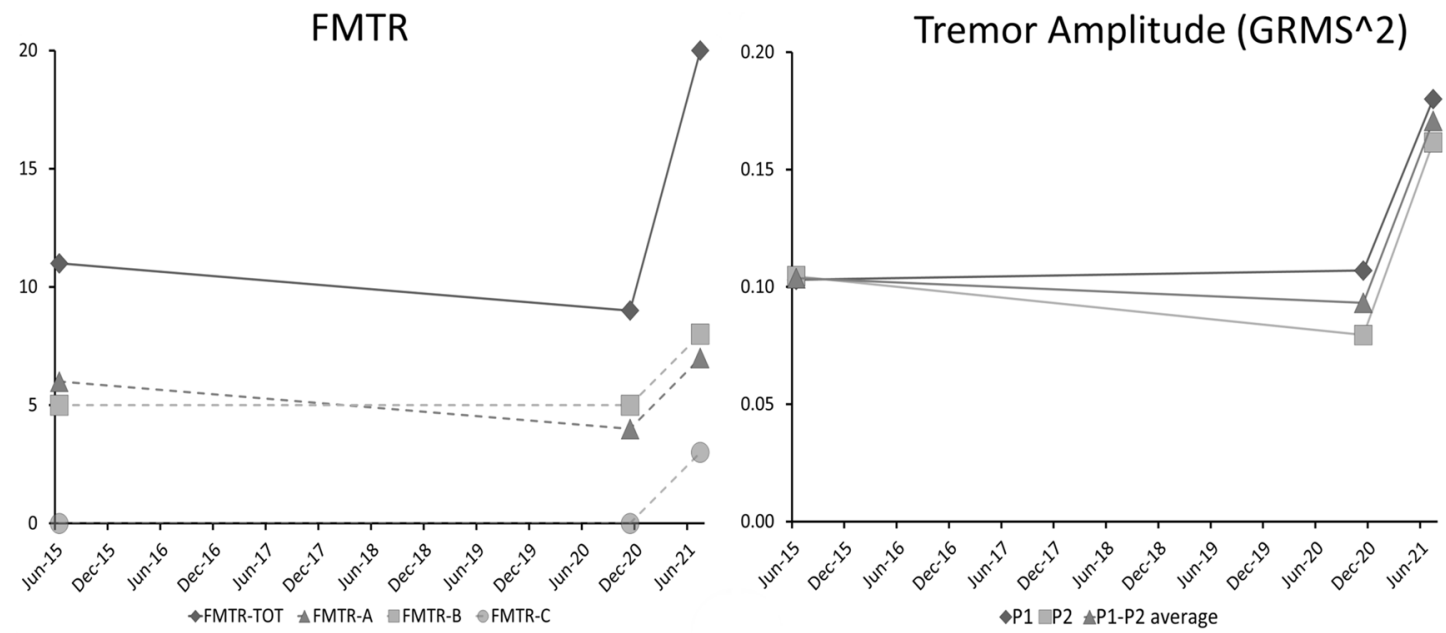

Drawing $\mathrm{T} 1$

Drawing $\mathrm{T} 2$

Drawing T3
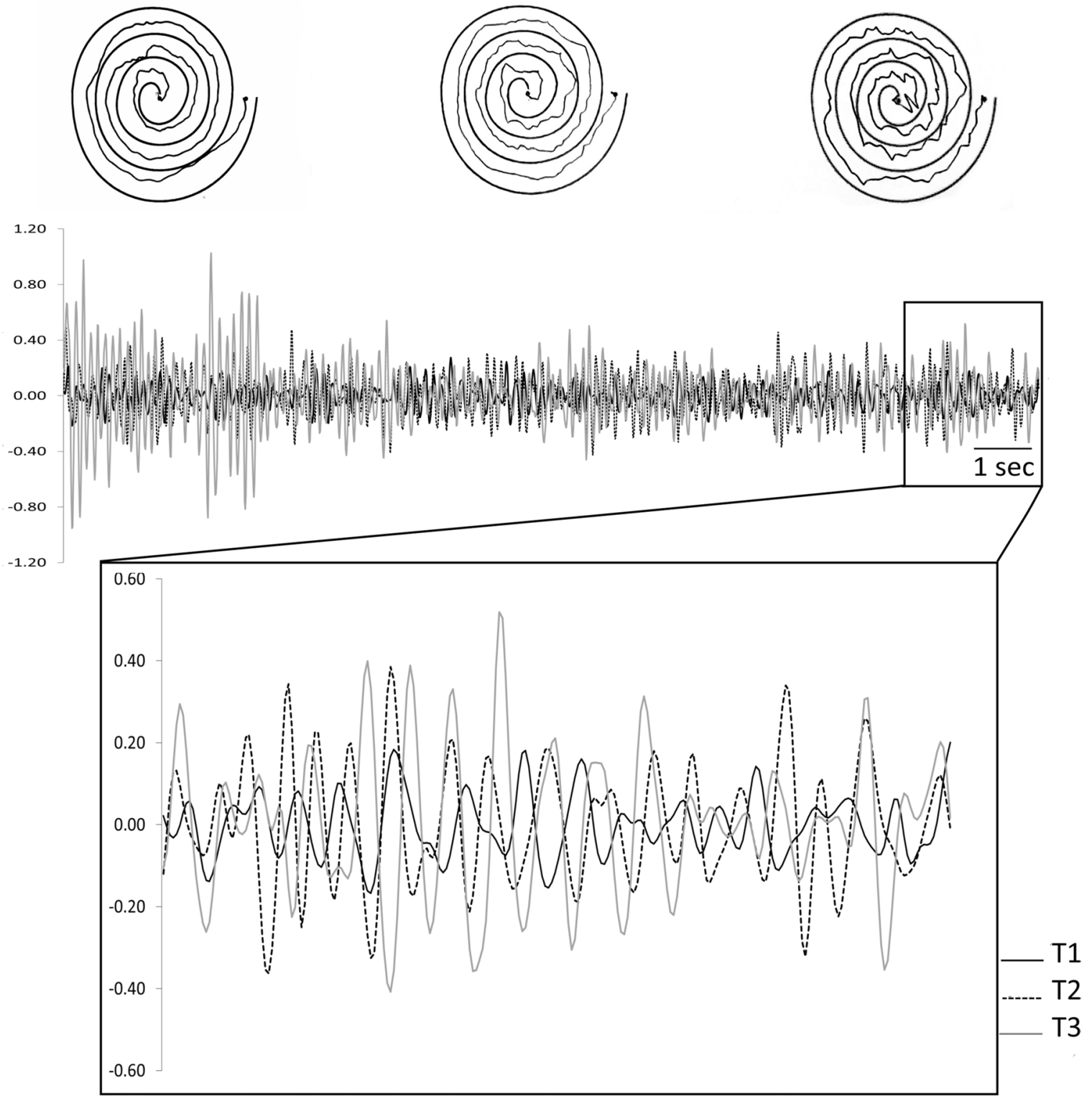
४Fig. 1 Upper part, left side: Fahn-Tolosa-Marin Tremor Rating Scale (FTMTRS) scores at T1 (June 2015), T2 (December 2020, before SARS-CoV-2 infection), and T3 (July 2021, after SARS-CoV-2 infection). Diamonds indicate total FTMTRS scores; triangles indicate section A subscores; squares indicate section B subscores; circles indicate section $\mathrm{C}$ subscores. Upper part, right side: postural tremor amplitude (average from the right and left upper limbs), expressed in terms of root mean square (RMS) of the acceleration traces of the reference marker in 3D space (GRMS^2), as kinematically assessed at $\mathrm{T} 1, \mathrm{~T} 2$, and $\mathrm{T} 3$, during posture 1 ( $\mathrm{P} 1)$ (arms outstretched, represented by diamonds) and posture 2 (P2) ("wing beating," represented by squares). Triangles indicate mean tremor amplitude values recorded during P1 and P2. Middle part: spiral drawing at T1, T2, and T3. Lower part: kinematic acceleration frames on the $y$-axis (15-s duration) recorded from the reflective marker placed on the left index finger of the patient while maintaining P1. Black solid lines represent kinematic recordings performed at $\mathrm{T} 1$. Black dashed lines and light gray lines represent kinematic recordings performed at $\mathrm{T} 2$ and $\mathrm{T} 3$ respectively

compared to T1 (posture 1: $+74 \%$; posture $2:+55 \%$ ) and T2 (posture 1: $+68 \%$; posture 2: 102\%) (Fig. 1), with no variation in tremor frequency and no difference in kinetic tremor between T1, T2, and T3. Dosage of propranolol administration was not increased because of a low-normal heart rate. No other medication was added. In September 2021, the patient was administered a single vaccine dose, no SARS$\mathrm{CoV}-2$ antibody title was acquired before. At clinical evaluation, in November 2021, tremor features were unchanged.

In summary, we report the case of an ET patient whose symptoms worsened after asymptomatic SARS-CoV-2 infection. Clinical tremor worsening was objectively confirmed by kinematic tremor analysis performed in comparison with the two previous evaluations. Tremor worsening was unlikely to be due to infection-related psychiatric and/ or cognitive status changes since clinical scores remained unchanged.

The development of transient or permanent movement disorders after viral infection is a well-established finding $[6,8]$ that has also been reported after SARS-CoV-2 infection $[2,3,5,6]$. Pre-infection neurological status represents a major issue in understanding whether a direct link exists between infection and neurological changes [3]. In the case of our patient, a 66-month follow-up (T1-T2) highlighted an objective stability of tremor severity over time. The worsening reported 7 months later (T2-T3) strongly suggested a relationship between tremor worsening and SARS-CoV-2 infection in ET.

Coronaviruses may have neuroinvasive potential in humans, spreading transneuronally to structures connected with the olfactory bulb [2]. Although direct invasion has been hypothesized $[2,3]$, there are no conclusive data to indicate that this is the major pathological mechanism of brain involvement in COVID-19 [2, 9]. In our case, we cannot exclude direct brain involvement since a negative brain scan has been reported in some cases of SARS-CoV-2-related encephalitis [9]. Moreover, we did not collect the patient's cerebrospinal fluid. Notably, the most comprehensive report on neuropathological findings from patients who died of COVID-19 showed a uniform presentation, with microglial activation and cytotoxic $\mathrm{T}$ cell lymphocytic infiltration [10]. In addition, the presence of SARS-CoV-2 in the brain was not associated with the severity of these neuropathological changes [10]. Interestingly, inflammatory findings associated with SARS-CoV-2 infection were mostly confined to the brainstem and cerebellum [10]. Dysfunction of the cerebello-thalamic network is well recognized in ET pathophysiology [11, 12]. In ET patients, brain pathology depicts several cerebellar changes, including Purkinje cell loss and axonal swelling $[11,12]$. Dysfunction in Purkinje cell GABAergic output to the dentate nucleus leading to increased cerebello-thalamic connectivity has also been demonstrated [11, 12]. Altogether, we may hypothesize that the tremor worsening in our patient resulted from further virus-induced immunemediated cerebellar circuit dysfunction. We did not title, however, serum auto-antibodies responsible for tremor autoimmune conditions (i.e., anti-DPPX, anti-GFAP, anti-mGLUR1).

In conclusion, this is the first study demonstrating that SARS-CoV-2 infection may cause tremor worsening in ET. Our careful pre-infection assessment showed a clear temporal relationship between infection and tremor worsening, which probably reflects a causal link. Tremor worsening in our case was likely due to virus-induced immune-mediated functional alterations in cerebellar networks. More observations are needed to better determine the relation between SARS-CoV-2 and ET in clinical practice.

Acknowledgements The authors wish to thank the patient for his participation in this research.

Author Contribution Study conception and design: Matteo Bologna. Material preparation, data collection, and analysis were performed by Massimiliano Passaretti, Alessandro De Biase, Giulia Paparella, Luca Angelini, Antonio Cannavacciuolo, and Donato Colella. The first draft of the manuscript was written by Massimiliano Passaretti and all authors reviewed the previous versions of the manuscript. All authors read and approved the final manuscript.

\section{Declarations}

Ethical Compliance Statement The authors confirm that the approval of an institutional review board was not required for this work. A written informed consent of the patient was obtained for the publication of his data. The authors have read the Journal's position on issues involved in ethical publication and affirm that this work is consistent with those guidelines.

Competing Interests The authors declare no competing interests. 


\section{References}

1. Huang C, Wang Y, Li X, Ren L, Zhao J, Hu Y, et al. Clinical features of patients infected with 2019 novel coronavirus in Wuhan, China. Lancet. 2020;395:497-506.

2. Scoppettuolo P, Borrelli S, Naeije G. Neurological involvement in SARS-CoV-2 infection: a clinical systematic review. Brain Behav Immun Health. 2020;5:100094.

3. Merello M, Bhatia KP, Obeso JA. SARS-CoV-2 and the risk of Parkinson's disease: facts and fantasy. Lancet Neurol. 2021;20:94-5.

4. Antonini A, Leta V, Teo J, Chaudhuri KR. Outcome of Parkinson's disease patients affected by COVID-19. Mov Disord. 2020. https://doi.org/10.1002/mds.28104.

5. Ghosh R, Biswas U, Roy D, Pandit A, Lahiri D, Ray BK, et al. De novo movement disorders and COVID -19: Exploring the Interface. Mov Disord Clin Pract. 2021;8:669-80.

6. Xing F, Marsili L, Truong DD. Parkinsonism in viral, paraneoplastic, and autoimmune diseases. J Neurol Sci. 2021:120014. https://doi.org/10.1016/j.jns.2021.120014.

7. Paparella G, Angelini L, De Biase A, Cannavacciuolo A, Colella D, Di Bonaventura C, et al. Clinical and kinematic features of valproate-induced tremor and differences with essential tremor. Cerebellum. 2021;20:374-83.
8. Cucca A, Migdadi HA, Di Rocco A. Infection-mediated autoimmune movement disorders. Parkinsonism Relat Disord. 2018;46 Suppl 1:S83-6.

9. Siow I, Lee KS, Zhang JJY, Saffari SE, Ng A. Encephalitis as a neurological complication of COVID-19: a systematic review and meta-analysis of incidence, outcomes, and predictors. Eur J Neurol. 2021;28:3491-502.

10. Matschke J, Lütgehetmann M, Hagel C, Sperhake JP, Schröder AS, Edler C, et al. Neuropathology of patients with COVID19 in Germany: a post-mortem case series. Lancet Neurol. 2020;19:919-29.

11. Pietracupa S, Bologna M, Tommasin S, Berardelli A, Pantano P. The contribution of neuroimaging to the understanding of essential tremor pathophysiology: a systematic review. Cerebellum. 2021. https://doi.org/10.1007/s12311-021-01335-7.

12. Haubenberger D, Hallett M. Essential tremor. Solomon CG, editor. N Engl J Med. 2018;378:1802-10.

Publisher's Note Springer Nature remains neutral with regard to jurisdictional claims in published maps and institutional affiliations. 